\title{
Recurrent Neural Approaches for Power Transformers Thermal Modeling
}

\author{
Michel Hell ${ }^{1}$, Luiz Secco ${ }^{2}$, Pyramo Costa Jr. ${ }^{2}$, and Fernando Gomide ${ }^{1}$ \\ 1 State University of Campinas - UNICAMP, \\ Av. Albert Einstein, 400, 13083-852, Campinas, SP - Brazil, \\ \{mbhell, gomide\}@dca.fee.unicamp.br \\ 2 Pontifical Catholic University of Minas Gerais - PUC-MG,
} Av. Dom José Gaspar, 500, Prédio 3, 30535-610, Belo Horizonte, MG - Brazil pyramo@pucminas.br

\begin{abstract}
This paper introduces approaches for power transformer thermal modeling based on two conceptually different recurrent neural networks. The first is the Elman recurrent neural network model whereas the second is a recurrent neural fuzzy network constructed with fuzzy neurons based on triangular norms. These two models are used to model the thermal behavior of power transformers using data reported in literature. The paper details the neural modeling approaches and discusses their main capabilities and properties. Comparisons with the classic deterministic model and static neural modeling approaches are also reported. Computational experiments suggest that the recurrent neural fuzzy-based modeling approach outperforms the remaining models from both, computational processing speed and robustness point of view.
\end{abstract}

\section{Introduction}

Power transformers are essential to transmit and distribute electric energy. Transformer faults may deeply affect power systems, causing power outages and socio-economic losses. Condition monitoring of power transformers is a key issue to guarantee safe operation, power availability, and service life.

A major factor in power transformer operation concerns its working temperature, that is, its hot-spot temperature. The hot-spot temperature has a vital role in insulation aging and service life of power transformers. Monitoring of hot-spot temperature is imperative to develop overload protection systems. High hot-spot temperature also means increasing rate of aging of transformers.

A central element to monitor power transformers condition is dynamic models to describe their thermal behavior. Differences between measured and predicted working temperature provide key information on transformer conditions and may indicate potential abnormalities.

Transient heat equations and specific thermal characteristics constitute an important approach to characterize the thermal capability of power transformers [1]. Conventional modeling techniques are useful to estimate internal temperatures of power transformers, but they still pose substantial challenges. Frequently, security encourages adoption of conservative safety factors. In practice this means underutilized transformers since hot-spot parts of the coils are kept from overheating and failing prematurely. In general 
the maximum power transfer found are $20-30 \%$ lower than the nominal power transformer capacity [2]. To bring power transformers beyond their nominal specifications to increase system operational margin during overload periods requires new loading schemes.

Among the different modeling techniques available today, artificial neural networks and fuzzy systems are examples that have been shown to be relevant and efficient in many application domains. The reason why neural, fuzzy models, and their hybridizations are extensively used in practice concerns their ability to learn complex non-linear relationships and to naturally treat imprecise data, a situation that is much more difficult to handle using conventional approaches [3]. An alternative that has received considerable attention, and shown significant progress in the recent years, is the use of recurrent connections in artificial neural networks and fuzzy models. In recurrent models, feedback connections introduce dynamics and the model become sensitive to the history of input data, an essential ingredient in modeling dynamic systems [3].

This work addresses two different recurrent neural models to model the thermal condition of power transformers. The first is the Elman recurrent neural network (ELM) [6] and the second is a recurrent neural fuzzy network (RNF) suggested by Ballini and Gomide [7]. The paper describes both neural modeling approaches and summarizes their main capabilities and properties. The models have been tested using actual data reported in the literature. Comparisons of these models with the deterministic [1] and static neural networks models are also included.

\section{Recurrent Neural Model - ELM}

We first review he recurrent neural model introduced by Elman [6]. The ELM is attractive to model dynamic systems because they are capable to learn temporal input/output relationships between patterns.

The structure of Elman neural network is composed by four layers: input layer, hidden layer, context layer, and output layer, as depicted in Figure 1 Learning is performed adjusting the weights connecting the neighboring layers. The self (recurrent) connections of the context nodes turn the network sensitive to the history of input/output data.

If the network inputs are $u(t) \in \mathbb{R}^{m}, y(t) \in \mathbb{R}^{n}, x(k) \in \mathbb{R}^{r}$, the outputs of each layer are found using:

$$
\begin{aligned}
& x_{j}(t)=f\left(\sum_{i=1}^{m} w 1_{i j} u_{i}(t)+\sum_{l=1}^{r} w 3_{l j} c_{l}(t)\right) \\
& c_{l}(t)=x_{j}(t-1) \\
& y_{q}(t)=g\left(\sum_{j=1}^{r} w 2_{j q} x_{j}(t)\right)
\end{aligned}
$$

where $w 1_{i j}$ is the weight connecting node $\mathrm{i}$ in the input layer to node $\mathrm{j}$ in the hidden layer, $w 2_{j q}$ is the weight connecting node $\mathrm{j}$ in the hidden layer with node $\mathrm{q}$ in the output layer, $w 3_{l j}$ is the weight connecting context node 1 to node $\mathrm{j}$ in the hidden layer, and $m, n, r$ are the number of nodes in the input, output, and hidden layers, respectively. $u_{i}(t), y_{j}(t)$ are the inputs and outputs, with $i=1, \cdots, m$ and $j=1, \cdots, n, x_{i}(t)$ is the 


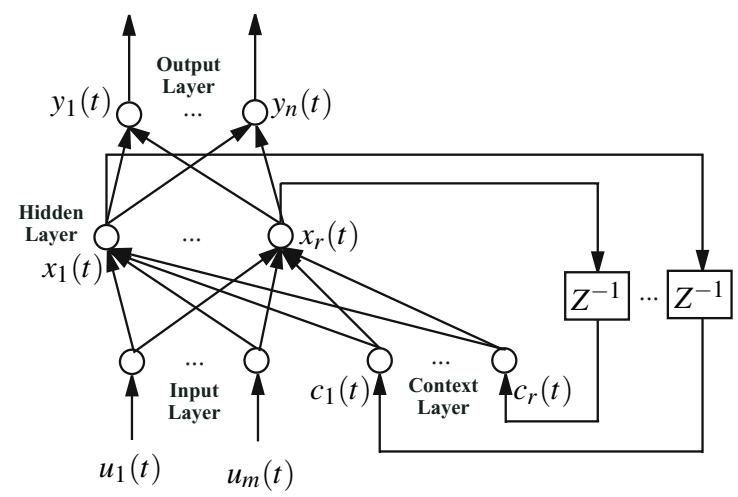

Fig. 1. Structure of the Elman network

output of the hidden node $\mathrm{i}, i=1, \cdots, r, c_{i}(t)$ the output of the context node i, i.e., the output of the hidden node $\mathrm{i}$ at $t, Z^{-1}$ the unit delay operator, $f(\bullet)$ and $g(\bullet)$ are activation functions of hidden layer and output layer neurons, respectively.

\section{Recurrent Neural Fuzzy Model - RNF}

The structure of the recurrent neural fuzzy network consists of two neural systems. The first has an input and two hidden layers and performs fuzzy inference. The second is a classic neural network that acts as operator to aggregate the outputs of the fuzzy inference system (Figura 2). This structure and learning procedure were initially introduced in [7]. Below we detail processing and learning scheme the recurrent neural fuzzy network addressed in this paper.

The first hidden layer consists of neurons whose activation functions are membership functions of fuzzy sets that defines the input space granulation. That is, for each

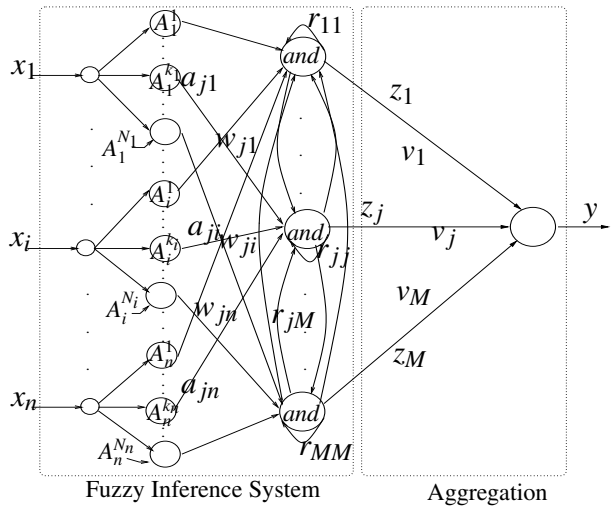

Fig. 2. RNF Model Structure

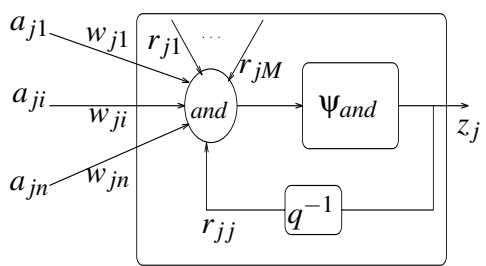

Fig. 3. Recurrent Fuzzy Set-Based Neuron 
dimension $x_{i}(t)$ of a $n$-dimensional input vector $\mathbf{x}(t)$ there are $N_{i}$ fuzzy sets $A_{i}^{k_{i}}, k_{i}=$ $1, \ldots, N_{i}$ whose membership functions are activation functions of corresponding input layer neurons. The variable $t$ is the discrete-time, that is, $t=1,2, \ldots$, but it will be omitted from now on to simplify notation. The outputs of the first hidden layer are the membership degrees of the input values, $a_{j i}=\mu_{A_{i}^{k_{i}}}\left(x_{i}\right), i=1, \ldots, n$ and $j=1, \ldots, M$; where $M$ is the number of neurons in the hidden layer.

Neurons of the second hidden layer are fuzzy set-based neurons, namely recurrent and neurons (Figure 3) with inputs $a_{j i}$ weighted by $w_{j i}$ and feedback connections weighted by $r_{j l}, l=1, \ldots, M$. Notice that fuzzy set-based neurons use triangular norms $(t$-norms and $s$-norms)to process its inputs. Characterization and different instances of $t$-norms and $s-$ norms are discussed in [5] and references therein. Processing steps of the neural fuzzy network are as follows:

1. $N_{i}$ is the number of fuzzy sets that composes the partition of $i$-th input;

2. $a_{j i}=\mu_{A_{i}^{k_{i}}}\left(x_{i}\right)$ is the membership degree of $x_{i}$ in the fuzzy set $A_{i}^{k_{i}}$, where $a_{j i}$ is the input of neuron $j$ of the second hidden layer;

3. $z_{j}$ is the $j-$ th output of the second hidden layer:

$$
z_{j}=\underset{i=1}{n+M}\left(w_{j i} \mathbf{s} a_{j i}\right)
$$

4. $y$, the output of nonlinear neuron, is given by:

$$
y=\psi(u)=\psi\left(\sum_{j=1}^{M}\left(v_{j} \cdot z_{j}\right)\right)
$$

where, $\psi: \mathfrak{R}^{M} \rightarrow[0,1]$ is a nonlinear, monotonically increasing function. In this paper, we use the logistic function $\psi(u)=1 /(1+\exp (-u))$;

5. $w_{j i}$ is the weight between the $j-$ th and-neuron and the $i-$ th neuron of the first hidden layer;

6. $v_{j}$ is the weight between the output $y$ of the network and the $j$-th neuron of the second hidden layer;

7. $r_{j l}$ is the weight of the feedback connection between the $j$-th and-neuron and the $l$-th neuron of the same, second hidden layer;

Note that the neural fuzzy network is quite flexible once it allows choosing appropriate triangular norms, and allows extraction of linguistic fuzzy rules directly from its topology, the fuzzy inference system part.

\section{Simulation Results}

This section addresses the performance of the recurrent neural models described in the previous section when they are used to estimate the hot-spot temperature of power transformers. For this purpose, we adopt the same data reported in [2]. Comparisons are made considering the recurrent neural models, the deterministic model suggested in [1], 
and three static (non-recurrent) neural models: multi layer perceptron network (MLP), radial basis function network (RBF), and neural fuzzy network (SNF) described in [5].

All five models were trained using measurements of the hot-spot temperature during 24 hours and a sampling period of 5 minutes [2]. The inputs of the neural models are the load currents $(K(t)),(K(t-1))$, and the top oil temperature $\left(T_{T O}(t)\right)$. The output is the hot-spot temperature $\left(T_{H}(\mathrm{t})\right)$.

After learning, the approximation, generalization and robustness capability of the neural models were verified using two data sets, each set describing different load capability conditions (with and without overload) as those embedded in the learning data of [2]. Test data were collected in the same way as training data.

Table 1 shows the values of performance index obtained after the simulation experiments. In Table $1, M S E-D t 1$ is the mean square error (MSE) obtained after models are run with test data with no overload condition. $M S E-D t 2$ is the MSE obtained when models process test data with overload. MSE - Dts is the MSE obtained when models simultaneously process both data sets.

Table 1. Simulation Results

\begin{tabular}{|c|c|c|c|c|}
\hline Model & Learning Time (seg.) & MSE-Dt1 & MSE-Dt2 & MSE-Dts \\
\hline MD & - & 17,3489 & 6,7434 & 12,0462 \\
\hline MLP & 92,76 & 0,7900 & 2,4880 & 1,6390 \\
\hline RBF & 82,63 & 0,2565 & 0,9914 & 0,6239 \\
\hline SNF & 61,50 & 0,2689 & 1,1025 & 0,6857 \\
\hline ELM & 59,39 & 0,4780 & 2,1069 & 1,2925 \\
\hline RNF & 31,37 & 0,0481 & 0,2635 & 0,1557 \\
\hline
\end{tabular}

As Table 1 suggests, all neural models satisfactorily estimate hot-spot temperature values. Note, however, that when training data contains spurious data, that is, noisy values not consistent with the actual behavior (non ideal training data), recurrent models become more attractive than static models. Recurrent models are more precise to capture transformer dynamics and gives more robust estimates of the hot spot temperature.

Table 2. Results using non ideal training data

\begin{tabular}{|c|c|c|c|c|c|c|}
\hline & MD & MLP & RBF & SNF & ELM & RNF \\
\hline MSE & 6,7434 & $1,2487 \times 10^{2}$ & $4,6579 \times 10^{4}$ & $0,2804 \times 10^{2}$ & 1,8986 & 0,7912 \\
\hline
\end{tabular}

To further investigate this important issue, we deliberately use a data set that does not fully represent the transformer dynamics to train the neural models. More specifically, we assume data with no overload conditions during training and data with overload to test approximation, robustness and generalization capabilities. Figure 4 depicts the estimates of the hot-spot temperature given by each model. Table 2 shows the mean square approximation errors. Interestingly, static models were unable to keep the same 

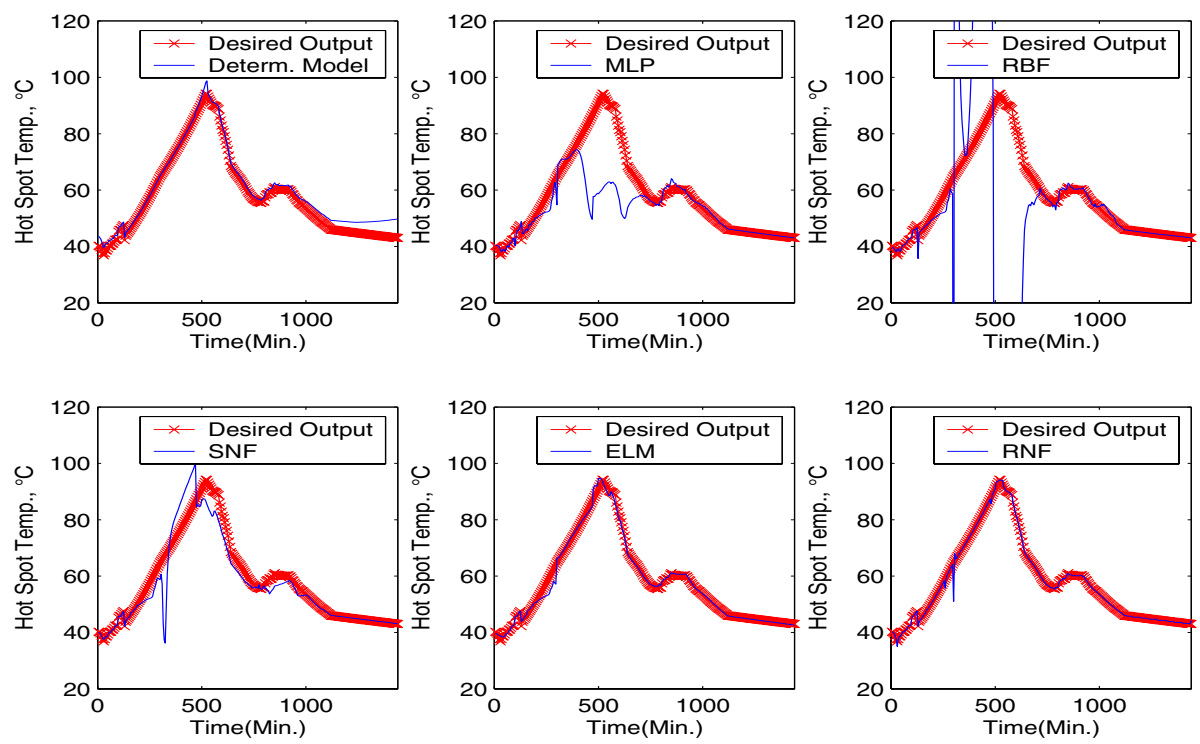

Fig. 4. Robustness Avaliation

performance when dealing with ideal training data while recurrent models did keep the same prediction performance as before. We also note that the recurrent neural fuzzy network - RNF - performs better, learns faster, and is more robust than- ELM.

\section{Conclusion}

In this work different neural approaches have been investigated as models for the thermal behavior of power transformers. Simulation experiments indicate that recurrent models are more robust than static neural models, especially when non-ideal data set is used during learning. Recurrent models capture the input/output relationship, their temporal relationship, and are more robust to noisy training data. Between the two recurrent models, the recurrent fuzzy neural network outperformed the Elman network. The recurrent fuzzy neural network offers an effective and robust power transformer model, learns quickly, runs fast, and needs modest computational requirements.

\section{Acknowledgment}

The first author acknowledges FAPESP, the Research Foundation of the State of São Paulo, for fellowship 03/05042-1, and the last thanks CNPq, the Brazilian National Research Council, for grant 475295/2003-0. The remaining authors also thank the Pontifical Catholic University of Minas Gerais (IPUC, PUC-MG) for its support. The authors are also in debt wit Álvaro Martins and Ronald Moura, from the Energetic Company of Minas Gerais (CEMIG), for their continuous help and motivation during the development of this work. 


\section{References}

1. G. W. Swift et. al., Adaptative Transformer Thermal Overload Protection, IEEE Transactions on Power Delivery, Vol. 16, No. 4, pp. 516-521, 2001.

2. V. Galdi, L. Ippolito, A. Piccolo and A. Vaccaro, Neural Diagnostic System for Transformer Thermal Overload Protection, IEE Proceedings of Electric Power Applications, Vol. 147, No. 5, pp. 415-421, 2000.

3. K.S. Narendra, K. Parthasarathy, Identification and Control of Dynamic Systems using Neural Networks, IEEE Transactions on Neural Networks, Vol. 1, pp. 4-27, 1990.

4. S. Haykin, Neural Networks: A Comprehensive Foundation, Prentice Hall, NJ-USA, ed. 2, 1998.

5. J.-S. R. Jang, ANFIS: Adaptative-Network-Based Fuzzy Inference System, IEEE Transactions on System, Man, and Cybernetics, Vol. 23, No. 3, 665-685, 1993.

6. J. Elman, Finding Structure in Time, Cognitive Science, Vol. 14, pp. 179-211, 1990.

7. R. Ballini and F. Gomide, Learning in recurrent, hybrid neurofuzzy networks, IEEE International Conference on Fuzzy Systems, pp. 785-791, 2002. 